\title{
Lamb Wave Dispersion Compensation in Piezoelectric Wafer Active Sensor Phased-Array Applications
}

\author{
Buli Xu, Lingyu Yu, Victor Giurgiutiu \\ Mechanical Engineering Department, University of South Carolina \\ Columbia, SC 29208 \\ xub@engr.sc.edu, yu3@engr.sc.edu, giurgiut@engr.sc.edu
}

\begin{abstract}
Lamb-wave testing for structural health monitoring is complicated by the dispersion nature of the wave modes. The dispersion effect will result in a propagated wave with longer time duration, deformed envelop shape as compared to its excitation counterpart, and hard to be interpreted. This paper first reviews the dispersion compensation and removal algorithms. Second, it compares these two methods by applying them to two widely used low-frequency Lamb wave modes: S0 and A0. Numerical simulations are compared in parallel with experimental results. Finally, the dispersion compensation algorithm is applied to 1-D PWAS phased array and demonstrated to improve the phase array's spatial resolution.
\end{abstract}

Keywords: Dispersion compensation, Lamb wave, Piezoelectric wafer active sensors, structural health monitoring, PWAS, SHM, PWAS phased array

\section{INTRODUCTION}

Lamb-wave testing for SHM is complicated by the dispersion nature of the wave modes. Dispersive waves have frequency-dependant propagation characteristics (Sachse and Pao, 1978). Hence, even narrowband Lamb wave excitations, such as tone bursts, will disperse as they propagate through structures. The dispersion effect will result in a propagated wave with longer time duration and deformed envelop shape as compared to its excitation counterpart. This deteriorates the wave spatial resolution and makes it hard to interpret the experimental data.

This dispersion issue can be addressed, for example, by using Lamb wave tuning technique (Giurgiutiu 2005, Santoni et al. 2007), which utilizes narrowband excitation and tune excitation frequency until a quasi non-dispersive Lamb wave mode is obtained. Alternatively, it can be addressed by using Lamb wave dispersion compensation algorithm proposed by Wilcox $(2001,2003)$. This algorithm makes use of a priori knowledge of the dispersion characteristics of a guided wave mode and performs signal processing algorithm to map signals from the time domain to the spatial domain and reverse the dispersion process. The basic idea underlying dispersion compensation is very similar to the time reversal procedure presented by $\mathrm{Xu}$ and Giurgiutiu (2007), in which a signal recorded at the receiver side is time reversed and propagated back to the source, the signal is then compressed to its original shape Instead of physically propagating back the received signal to its source, dispersion compensation method analytically maps the received signal back to its source to retrieve its original shape and location through signal processing approach. More recently, Liu and Yuan (2009) proposed a dispersion removal procedure based on the wavenumber linear Taylor expansion. By transforming a dispersed signal to frequency domain and interpolating the signal at wavenumber values satisfying the linear relation, the original shape of the signal can be recovered.

This paper first reviews the dispersion compensation and removal algorithms. Second, it compares these two methods by applying them to two widely used low-frequency Lamb wave modes: S0 and A0. Numerical simulations are compared in parallel with experimental results. Finally, the dispersion compensation algorithm is applied to 1-D PWAS phased array and demonstrated to improve the phase array's spatial resolution. 


\section{THEORY OF DISPERSION COMPENSATION AND REMOVAL}

\subsection{DISPERSED WAVE SIMULATION}

Figure 1 illustrates the use of PWAS in pulse-echo mode to detect crack damage in a thin-wall structure. PWAS transducer was bonded to the structure to achieve direct transduction of electric energy into elastic energy and vice-versa. In Figure 1, the compact wave $f(t)$ denotes the forward Lamb wave excitation; the elongated wave $g(t)$ denotes the dispersed Lamb wave reflected from the crack. The propagated $f(t)$ waveform at arbitrary spatial and temporal location $(x, t)$ can be predicted as

$$
u(x, t)=\frac{1}{2 \pi} \int_{-\infty}^{\infty} F(\omega) e^{j \omega(t-\alpha x)} d \omega=\frac{1}{2 \pi} \int_{-\infty}^{\infty} F(\omega) e^{j(\omega t-k x)} d \omega
$$

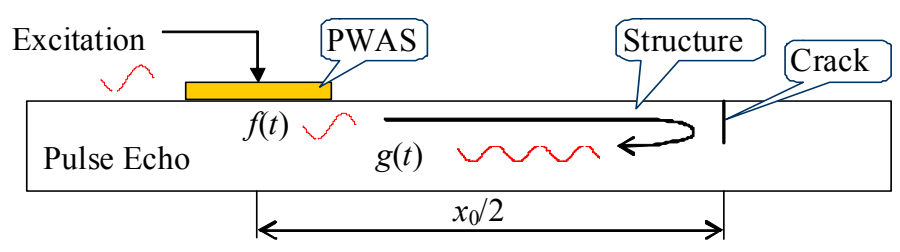

Figure 1 principles of pulse-echo method to detect a crack near PWAS transducers on a thin-wall structure

Assuming constant reflection coefficient $A$, and $x_{0} / 2$ distance separation between the PWAS and the crack; the reflected waveform after $x_{0}$ propagation distance can be predicted by

$$
g(t)=\left.u(x, t)\right|_{x=x_{0}}=\frac{A}{2 \pi} \int_{-\infty}^{\infty} F(\omega) e^{j\left(\omega t-k x_{0}\right)} d \omega
$$

Where, $F(\omega)$ is the Fourier transform of $f(t), k$ is the wavenumber. Eq. (2) enables us to simulate a dispersed wave after a certain propagation distance.

\subsection{DISPERSION COMPENSATION ALGORITHM}

If we propagate backward the $g(t)$ to its source location (i.e., set $t=0$ ) but in reversed propagation direction (i.e., set $x=-x$ ), we have dispersion compensated waveform $h(x)$ as

$$
h(x)=\left.u(x, t)\right|_{\substack{x=-x \\ t=0}}=\left.\frac{1}{2 \pi} \int_{-\infty}^{\infty} G(\omega) e^{j \omega(t-\alpha x)} d \omega\right|_{\substack{x=-x \\ t=0}}=\frac{1}{2 \pi} \int_{-\infty}^{\infty} G(\omega) e^{j k x} d \omega
$$

where $G(\omega)$ is the Fourier transform of $g(t)$. Note that Eq. (3) is the fundamental dispersion compensation equation. It maps the signal from time domain to spatial domain and reverses the dispersion process. Recall the definitions of group velocity $c_{g r}(\omega)$ and phase velocity $c_{p s}(\omega)$, we have

$$
\begin{aligned}
& d \omega=c_{g r}(\omega) d k \\
& \omega=c_{p h}(\omega) k
\end{aligned}
$$

Substitute (4) into (3), we have

$$
h(x)=\frac{1}{2 \pi} \int_{-\infty}^{\infty} H(k) e^{j k x} d k
$$

where, $H(k)=G(\omega) c_{g r}(\omega), \omega=\omega(k)$. The wavenumber $k$ can be thought of as a spatial frequency variable. Thus, $x$ and $k$ are dual variables for transforming between spatial and wavenumber domains, in the same sense as $t$ and $\omega$ are dual variables for transforming between time and frequency domains. Inverse Fourier transform methods (e.g., IFFT) can be used to calculate $h(x)$ in Eq. (5). However, besides interpolating $G(\omega)$ and $c_{g r}(\omega)$, careful setup of the variables in spatial/wavenumber domains w.r.t. those in time/frequency domains is needed to ensure the calculation accuracy. 


\subsection{DISPERSION REMOVAL ALGORITHM}

The Lamb wave nonlinear wavenumber $k(\omega)$ causes phase distortions and results in a dispersed waveform. If the wavenumber is in a linear relation w.r.t. $\omega$, there will be no dispersion in a propagated waveform. Based on this concept, the dispersion removal algorithm maps the signal to wavenumber domain where linear wavenumber relation is satisfied to removal the dispersion effect. The linear wavenumber relation is approximated by using the Taylor expansion of the wavenumber $k(\omega)$ at the excitation central frequency $\omega_{0}$ up to the first order as

$$
k(\omega) \approx k_{\text {lin }}(\omega)=k\left(\omega_{0}\right)+k^{\prime}\left(\omega_{0}\right)\left(\omega-\omega_{0}\right)=k\left(\omega_{0}\right)+\frac{\omega-\omega_{0}}{c_{g r}\left(\omega_{0}\right)}
$$

Therefore, to remove the dispersion in a dispersed wave, such as $g(t)$, the procedure can be summarized as follows:

- Apply Fourier transform to $g(t)$ and get $G(k)$, where $G(k)=G(\omega)$ since $k$ is a function of $\omega$;

- Use Eq. (6) to calculate linear wavenumber values $k_{\text {lin }}(\omega)$;

- Interpolate $[k, G(k)]$ pair at $k_{\text {lin }}(\omega)$ to get $G\left[k_{\text {lin }}(\omega)\right]$;

- Apply inverse Fourier transform to $G\left[k_{\text {lin }}(\omega)\right]$ to get a wave with the dispersion removed.

As compared to the dispersion compensation algorithm, the dispersion removal algorithm needs less computation efforts.

\section{DISPERSION COMPENSATION VERSUS DISPERSION REMOVAL}

Dispersion compensation and dispersion removal algorithms are compared in this section. The comparison is based on both numerical and experimental results for recovering two dispersed S0 and A0 Lamb waves

\subsection{NUMERICAL SIMULATION}

Numerical simulations comparison to recover the two widely used $S_{0}$ and $A_{0}$ modes on a 3-mm and 1 -mm aluminum plates $\left(\rho=2780 \mathrm{~kg} / \mathrm{m}^{3}, E=72.4 \times 10^{9} \mathrm{~Pa}\right)$ were performed. To maximize the dispersion effect of one mode while suppressing the other mode, tone burst excitations were selected to center at 350 $\mathrm{MHz}$ for $S_{0}$ mode on the 3-mm plate with group velocity $c_{g r}=5380 \mathrm{~m} / \mathrm{s}$ and at $36 \mathrm{kHz}$ for $A_{0}$ mode on the 1 -mm plate with group velocity $c_{g r}=1163 \mathrm{~m} / \mathrm{s}$

Simulation results of $S_{0}$ mode using dispersion compensation and removal algorithms are plotted in Figure 2. Figure 2a shows a Hanning windowed tone burst excitation centered at $350 \mathrm{kHz}$, which is used to excite $S_{0}$ mode Lamb wave. Figure $2 \mathrm{~b}$ illustrates the dispersed $S_{0}$ mode waveform after propagation distance of $300 \mathrm{~mm}$, simulated by Eq. (2). Because of dispersion, the waveform changes its envelope shape as it propagates through the structure. Figure 2c shows the waveform recovered by dispersion compensation algorithm using Eq. (5). Spatial resolution of the wave packet is largely improved as compared to its dispersed version. Meanwhile, the waveform is exactly sitting at the expected spatial location $x=300 \mathrm{~mm}$. Figure $2 \mathrm{~d}$ shows the recovered waveform by dispersion compensation algorithm and Figure 2e shows the recovered waveform by dispersion removal algorithm in time domain. Both of the recovered waveforms are very close to their original tone burst excitation. A close examination of these two recovered waveforms shows that dispersion removal algorithm show slightly better performance.

Similar simulation results, as shown in Figure 3, were observed when applying the dispersion compensation algorithm to a dispersed $36 \mathrm{kHz} A_{0}$ mode wave on a 1-mm aluminum plate. The spatial resolution of the dispersed $A_{0}$ mode wave was improved after mapping it from time domain to spatial domain using dispersion compensation algorithm. However, the compensated waveform in time domain (Figure 3d) seems to possess higher frequency components as compared to its original tone burst excitation. This may due to the artifacts introduced by mapping procedure (e.g., interpolation method) in 
dispersion compensation algorithm. In contrast, the recovered waveform by dispersion removal algorithm is very close to the shape of its original tone burst excitation, as shown in Figure 3d.

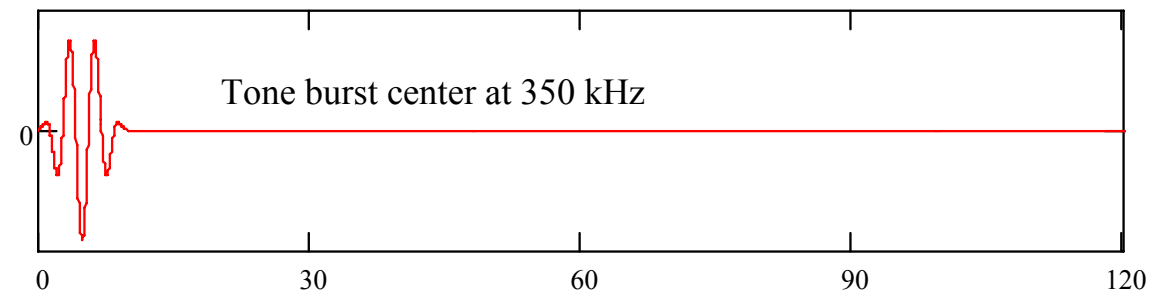

(a)

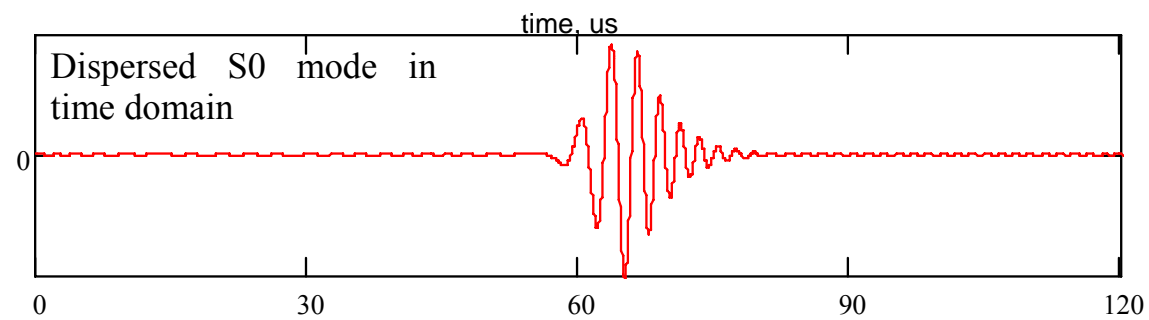

(b)

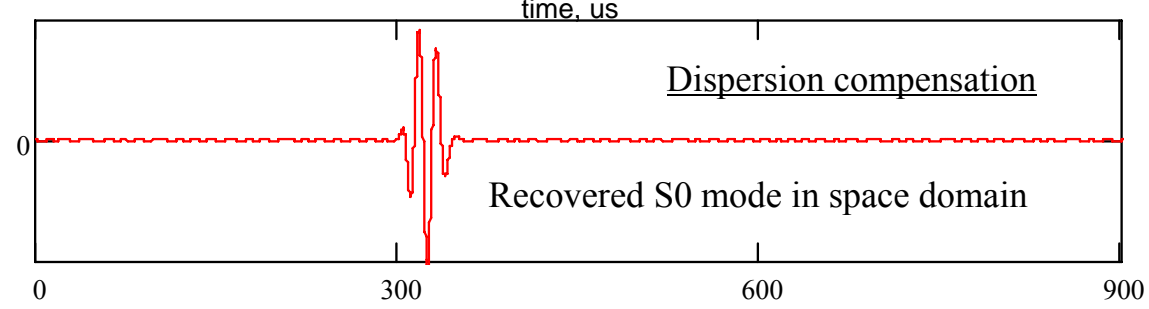

(c)

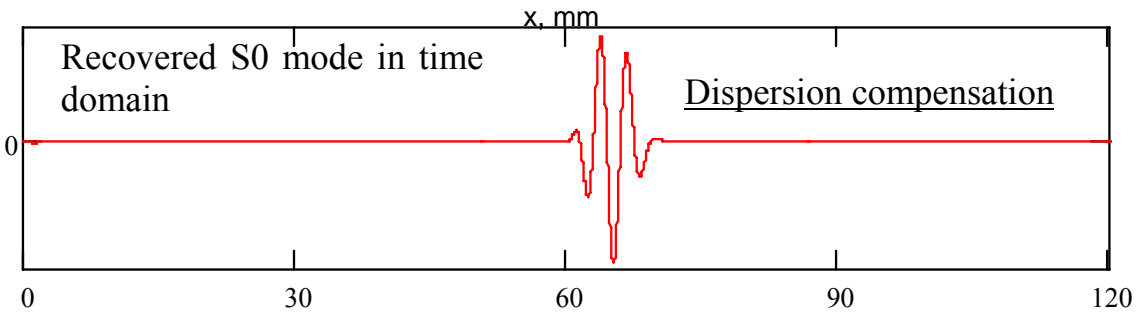

(d)

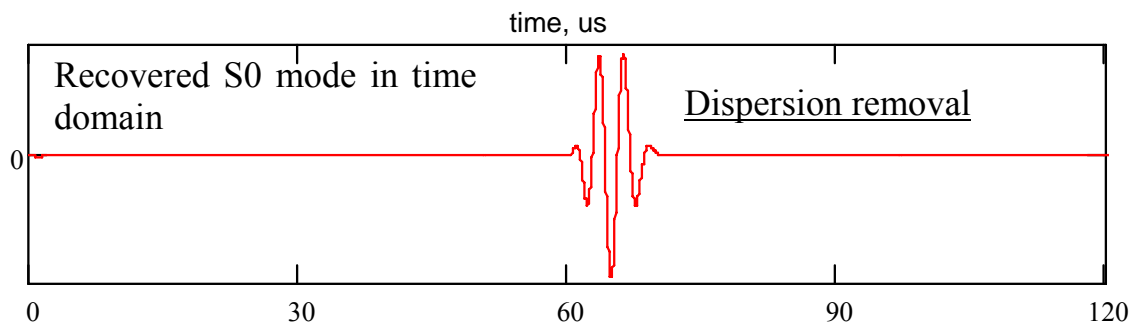

(e)

time, us

Figure 2 Numerical simulation of dispersion compensation of $350 \mathrm{kHz}$ S0 mode on a 3-mm aluminum plate: (a) 3.5-count Hanning windowed tone burst center at $350 \mathrm{kHz}$; (b) dispersed S0 mode wave after $x=300 \mathrm{~mm}$ propagation distance, simulated by Eq. (2); (c) recovered S0 mode wave in spatial domain by dispersion compensation algorithm; (d) recovered So mode wave in time domain by dispersion compensation algorithm; (e) recovered SO mode wave in time domain by dispersion removal algorithm 


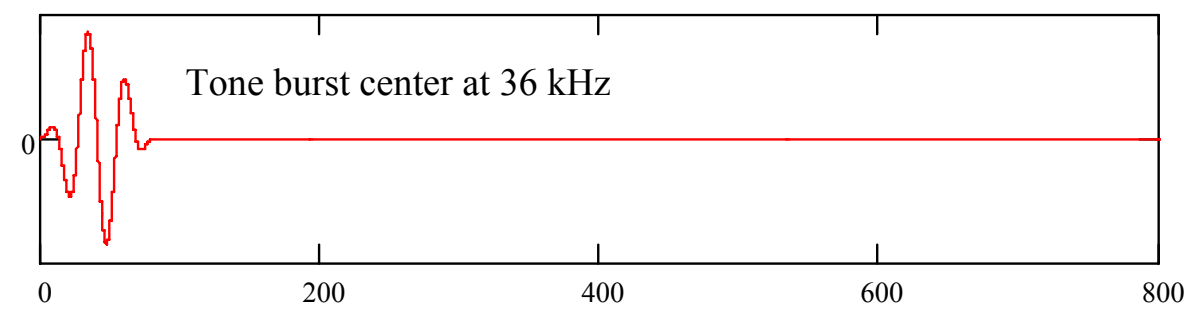

(a)

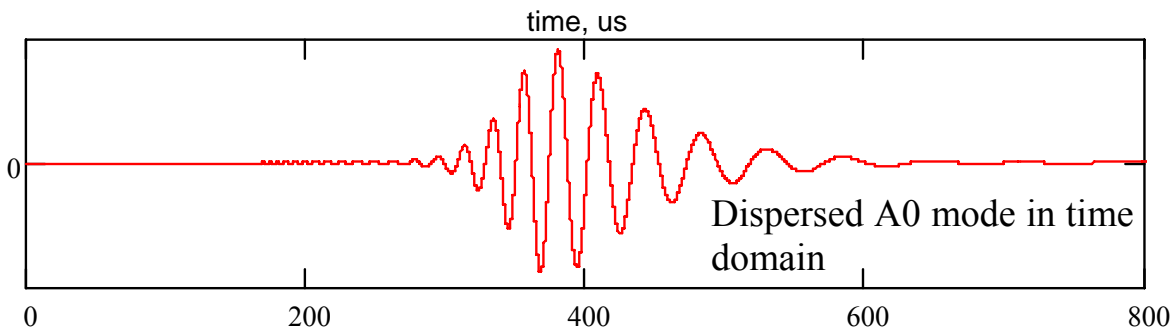

(b)

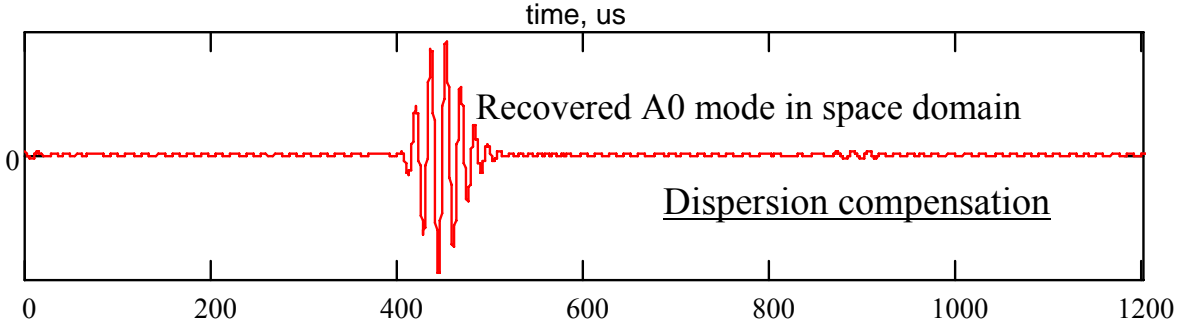

(c)

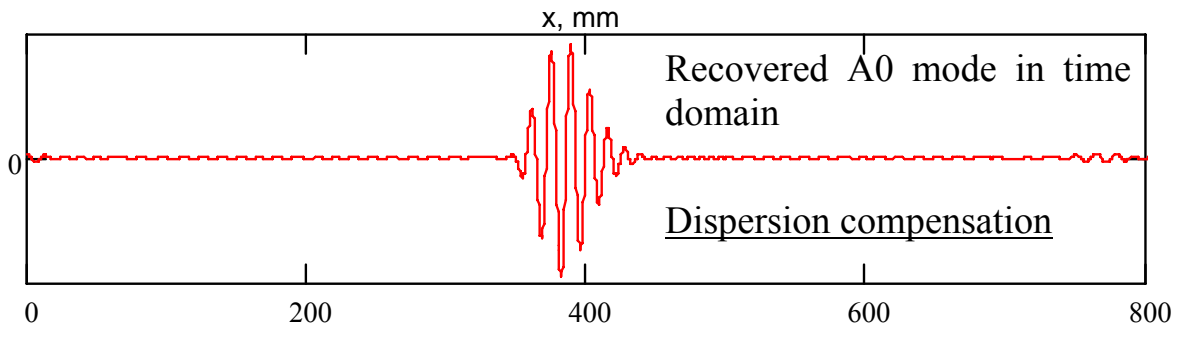

(d)

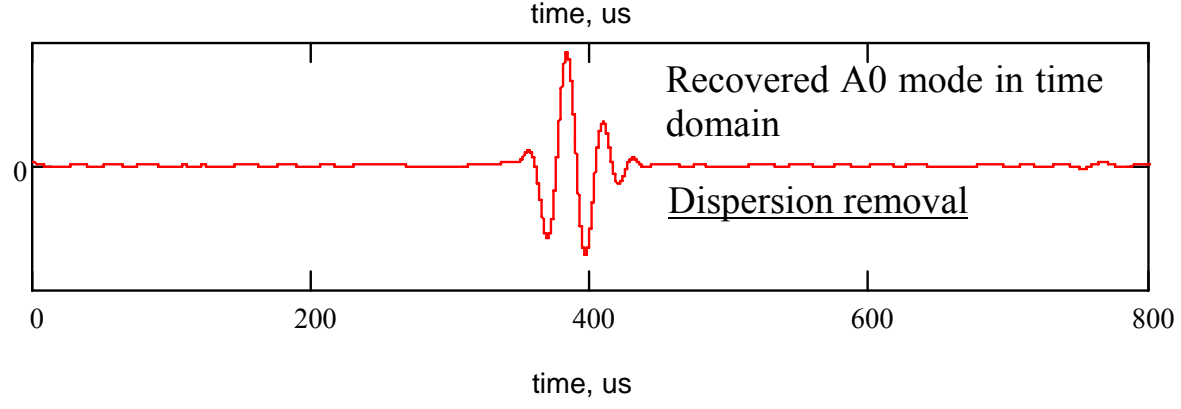

Figure 3 Numerical simulation of dispersion compensation of $36 \mathrm{kHz} \mathrm{A0}$ mode on a 1-mm aluminum plate: (a) 3-count Hanning windowed tone burst center at $36 \mathrm{kHz}$; (b) dispersed $\mathrm{A} 0$ mode wave after $x=400 \mathrm{~mm}$ propagation distance, simulated by Eq. (2); (c) recovered A0 mode wave in spatial domain by dispersion compensation algorithm; (d) recovered $A 0$ mode wave in time domain by dispersion compensation algorithm; (e) recovered A0 mode wave in time domain by dispersion removal algorithm 


\subsection{EXPERIMENTAL VERIFICATION}

\subsubsection{Experimental setup}

Figure 4 shows Lamb wave dispersion compensation and removal experimental setup using permanently bonded PWAS transducers. It consists of a HP33120 function generator, a Tektronix 5430B oscilloscope and a PC (Figure 4a). Two specimens were used: one is a $1524 \mathrm{~mm} \times 1524 \mathrm{~mm} \times 1 \mathrm{~mm}$ aluminum plate bonded with two round 7-mm diameter PWAS, $400 \mathrm{~mm}$ apart (Figure 4b); the other one is a $1060 \mathrm{~mm} \times 300 \mathrm{~mm} \times 3 \mathrm{~mm}$ aluminum plate bond with two 7-mm square PWAS, $300 \mathrm{~mm}$ apart (Figure $4 \mathrm{c}$ ). To eliminate boundary reflection interference, modeling clay was put around specimen edges. Instead of using one transmitter and one reflector working a pulse-echo mode, a pair of transducers working in pitch-catch mode was used for better SNR. All the signals from receivers were recorded by oscilloscope and saved in Excel data format.

\section{(a)}
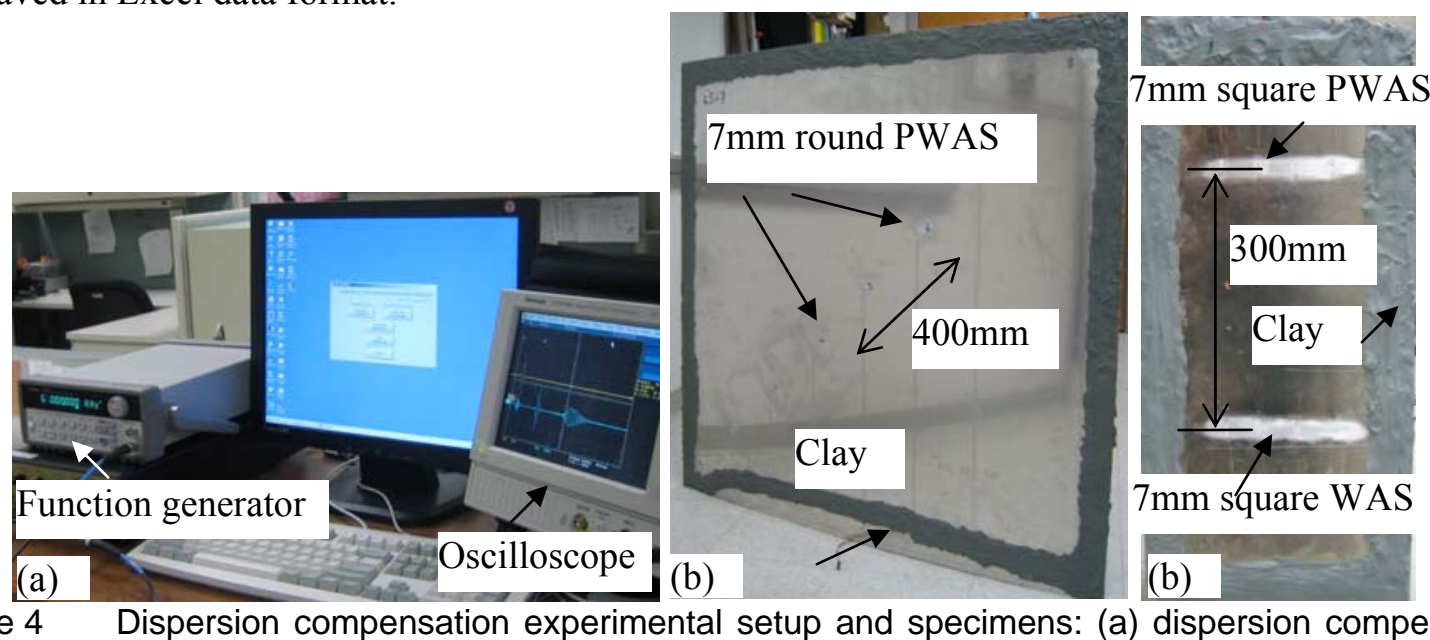

\subsubsection{Experimental results}

Before applying dispersion compensation and removal algorithms, the recorded waveforms need to be pre-processed, including DC removal, upsampling, and zero padding, to minimize frequency leakage and prevent Fourier transform data wrapping during the dispersion compensation algorithm.

Figure 5 shows the dispersion compensation and dispersion removal results of a $350 \mathrm{kHz}$ S0 mode wave on a 3-mm aluminum plate after propagation distance $x$ of $300 \mathrm{~mm}$. The experimental results are found to be very close to our numerical prediction. The dispersed S0 mode wave packets were well recovered by both algorithms.

Figure 6 shows the dispersion compensation and removal results of a $36 \mathrm{kHz} A_{0}$ mode wave on a 1$\mathrm{mm}$ aluminum plate after propagation distance $x$ of $400 \mathrm{~mm}$. The experimental results are very close to simulation results. Before applying dispersion compensation and removal algorithms, the A0 mode wave packet time span is around $200 \mu \mathrm{s}$. In contrast, the A0 mode wave packet was compressed to around 80 $\mu s$ span after dispersion compensation. The spatial resolution of A0 mode wave packet is largely increased after applying dispersion compensation and removal methods. Again, a high frequency component was observed in the recovered $\mathrm{A} 0$ when using dispersion compensation algorithm. 


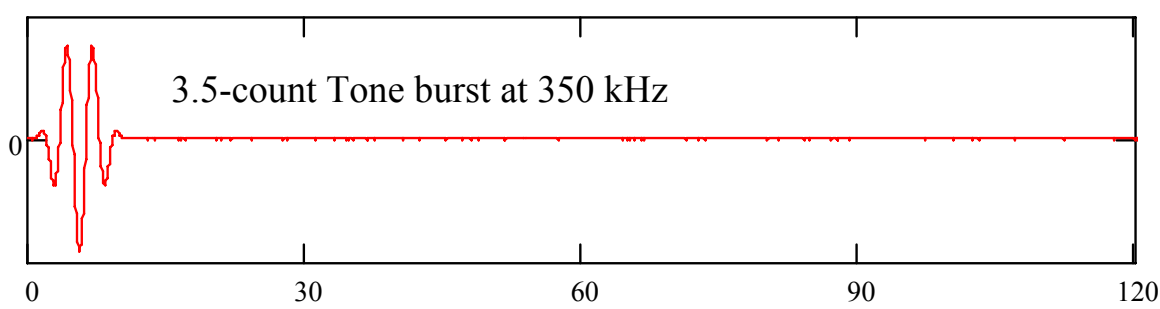

(a)

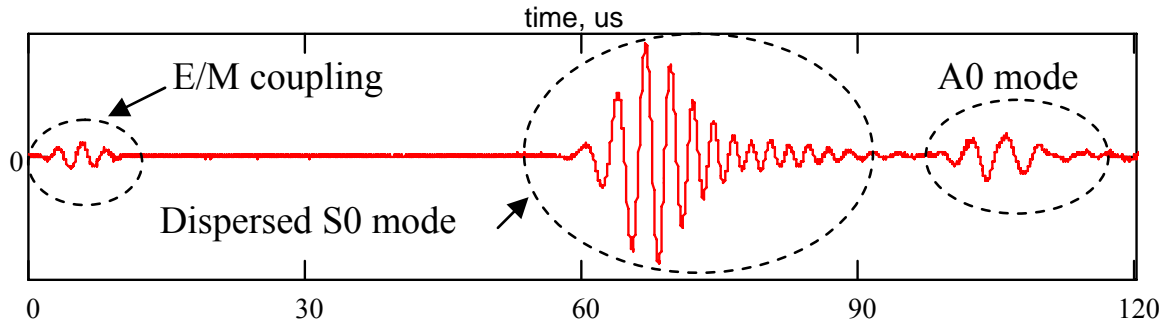

(b)

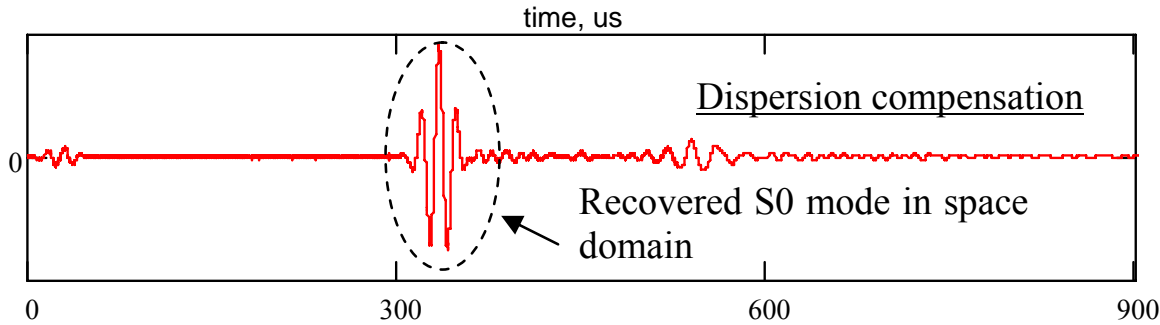

(c)

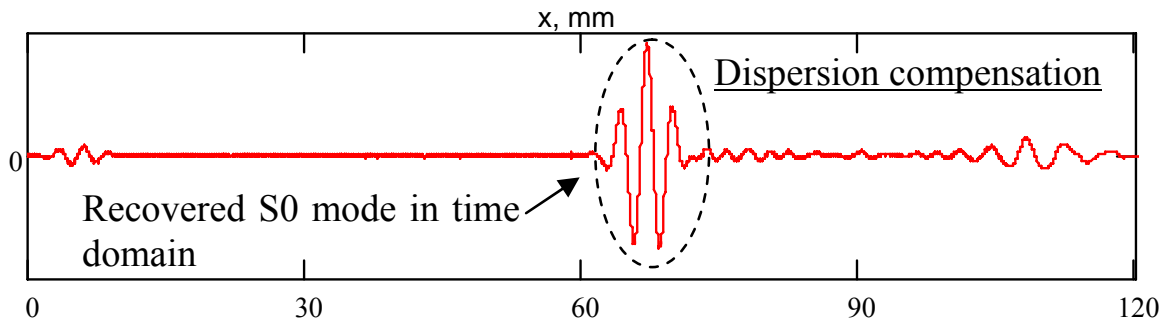

(d)

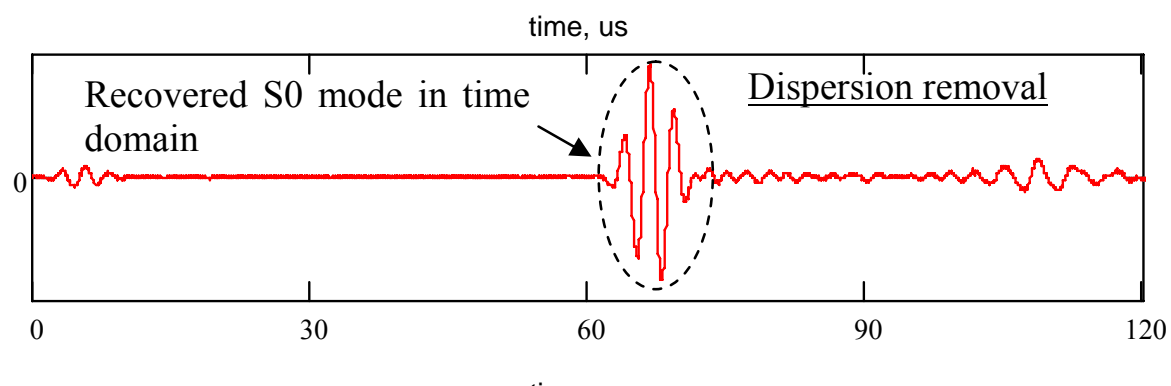

(e)

time, us

Figure 5 Experimental results of dispersion compensation of $350 \mathrm{kHz}$ SO mode on a 3-mm aluminum plate: (a) 3.5-count Hanning windowed tone burst center at $350 \mathrm{kHz}$; (b) dispersed S0 mode wave after $x=300 \mathrm{~mm}$ propagation distance, simulated by Eq. (2); (c) recovered S0 mode wave in spatial domain by dispersion compensation algorithm; (d) recovered SO mode wave in time domain by dispersion compensation algorithm; (e) recovered S0 mode wave in time domain by dispersion removal algorithm 


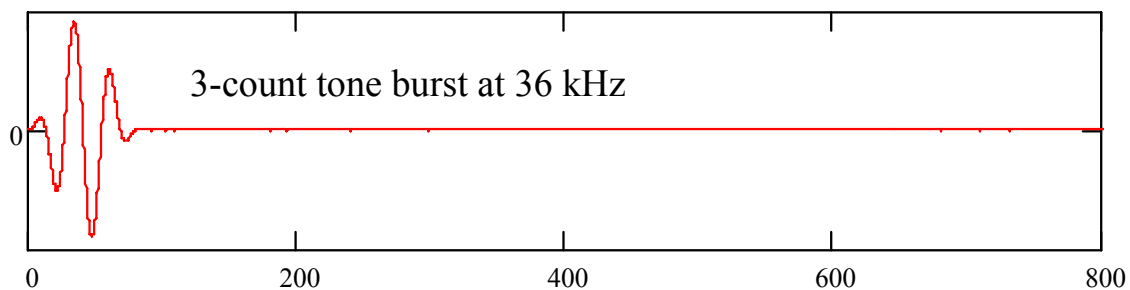

(a)

time, us

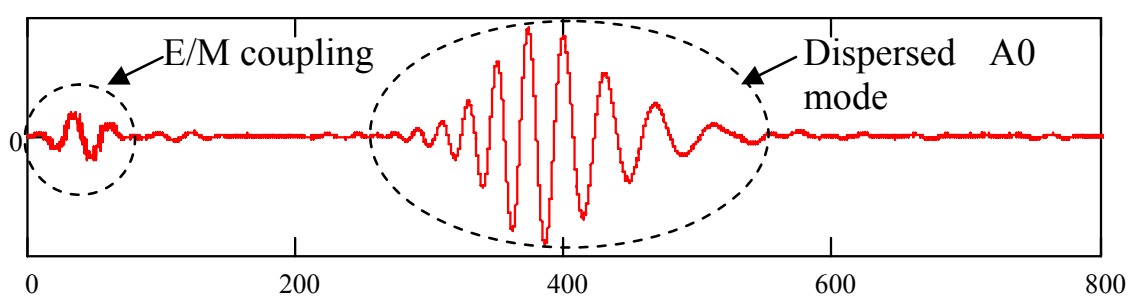

(b)

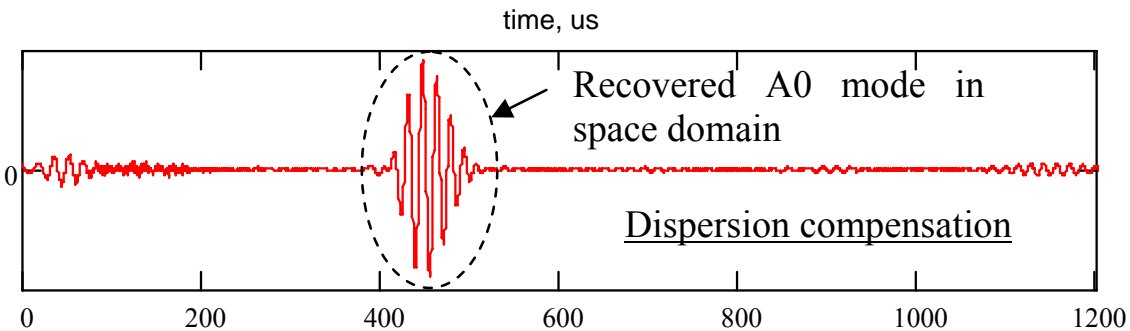

(c)

$\mathrm{X}, \mathrm{mm}$

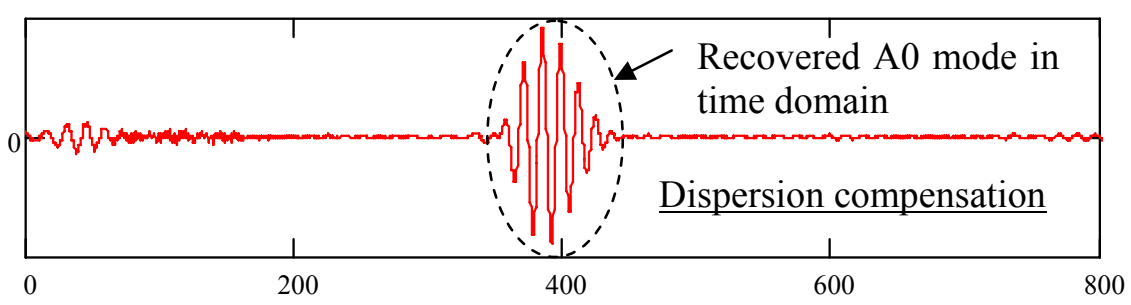

(d)

time, us

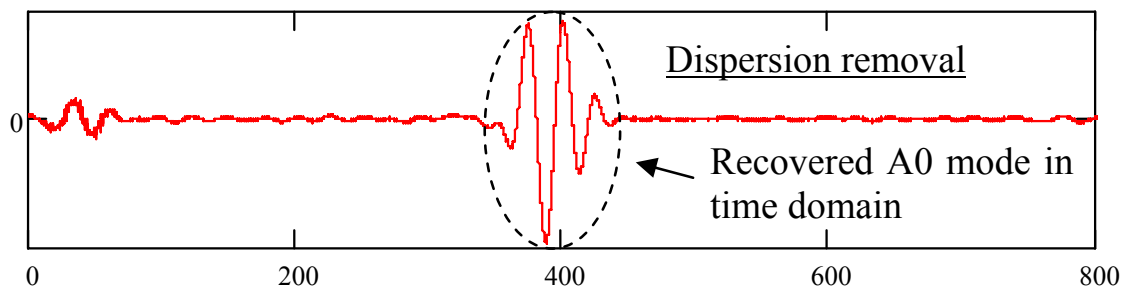

(e)

time, us

Figure 6 Experimental results of dispersion compensation of $36 \mathrm{kHz}$ A0 mode on a 1-mm aluminum plate: (a) 3-count Hanning windowed tone burst center at $36 \mathrm{kHz}$; (b) dispersed A0 mode wave after $x=400 \mathrm{~mm}$ propagation distance, simulated by Eq. (2); (c) recovered A0 mode wave in spatial domain by dispersion compensation algorithm; (d) recovered AO mode wave in time domain by dispersion compensation algorithm; (e) recovered A0 mode wave in time domain by dispersion removal algorithm 


\section{APPLICATION OF DISPERSION COMPENSATION TO PWAS PHASED ARRAY}

PWAS phased array uses a group of sensors located at distinct spatial locations and focuses the guided wave front in a specific direction by sequentially firing the individual elements in the array at different time. The beam steering and scanning is implemented using the embedded ultrasonic structural radar (EUSR) methodology in combination with the PWAS guided wave technology (Giurgiutiu and Bao, 2002) (Yu and Giurgiutiu, 2007). One requirement for the success of PWAS phased array, a single mode non-dispersive Lamb wave needs to be employed (Santoni et al. 2007). This largely limits the operation frequency selection of the phased array though the single mode situation has been solved by using the frequency tuning approach. In this section, we explored the application of dispersion compensation algorithm to 1-D linear PWAS array EUSR interrogation using tuned dispersive Lamb waves.

Recall that EUSR works in pulse-echo transducer mode and round-robin data collecting pattern. For an $M$-PWAS array, $M^{2}$ sets of signal data need to be collected. Assume, the $i^{\text {th }}$ PWAS is the transmitter supplied with tone burst excitation and the $j^{\text {th }}$ PWAS is the receiver. The received wave can be predicted by

$$
g_{i, j}(t)=\frac{A}{2 \pi} \int_{-\infty}^{\infty} T B(\omega) e^{j\left[\omega t-k\left(\left|\vec{r}_{i}\right|+\vec{r}_{j} \mid\right)\right]} d \omega \quad i, j=0,1, \ldots M-1
$$

where $T B(\omega)$ denotes tone burst excitation in frequency domain; $\vec{r}$ is transducer location vector. To simulate a $M=8$ PWAS array, 64 groups of source data will be generated. Each is saved in .csv data format. For EUSR without dispersion compensation, these 64 .csv files will be read directly and imaged by EUSR. In contrast, for EUSR with dispersion compensation, the 64 .csv files will first be processed by dispersion compensation algorithm and then imaged by EUSR. Four cases of 1-D PWAS phase array for damage detection were simulated, as shown in Table 4.1. Figure 7 shows the case I-IV aluminum plate and crack setup.

Table 4.1 Simulated cases of damage detection with 1-D PWAS phase array

\begin{tabular}{|l|l|l|l|l|l|}
\hline & $\begin{array}{l}\text { Lamb wave } \\
\text { freq. \& mode }\end{array}$ & $\begin{array}{l}\text { Group } \\
\text { velocity } \\
(\mathrm{m} / \mathrm{s})\end{array}$ & $\begin{array}{l}\text { Structure } \\
\text { (Plate) } \\
\text { thickness }\end{array}$ & $\begin{array}{l}\text { Crack location(s) } \\
y_{\text {crack }}(\mathrm{mm} / \mathrm{deg})\end{array}$ & $\begin{array}{l}\text { Sensor } \\
\text { spacing } \\
(\mathrm{mm})\end{array}$ \\
\hline Case I & 3 -count 36 kHz A0 & 1162 & $1-\mathrm{mm} \mathrm{Al}$ & $200 / 90^{\circ}$ & 8 \\
\hline Case II & 3.5 -count 350 kHz S0 & 5242 & 3 -mm Al & $150 / 90^{\circ}$ & 7 \\
\hline Case III & 3.5 -count 350 kHz S0 & 5242 & $3-\mathrm{mm} \mathrm{Al}$ & $150 / 90^{\circ}, 170 / 90^{\circ}$ & 7 \\
\hline Case IV & 3.5 -count 350 kHz S0 & 5242 & $3-\mathrm{mm} \mathrm{Al}$ & $150 / 90^{\circ}, 166 / 90^{\circ}$ & 7 \\
\hline
\end{tabular}

(a)

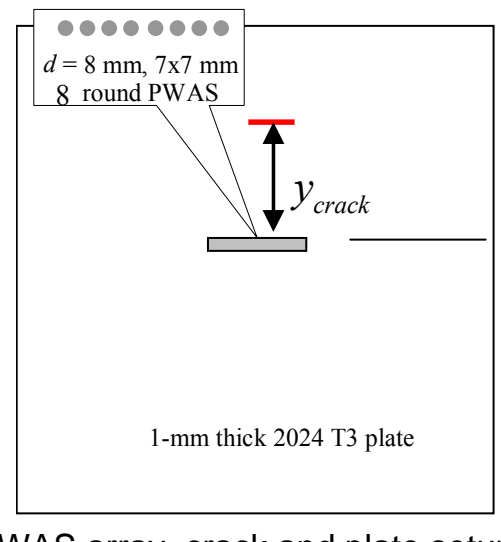

(b)

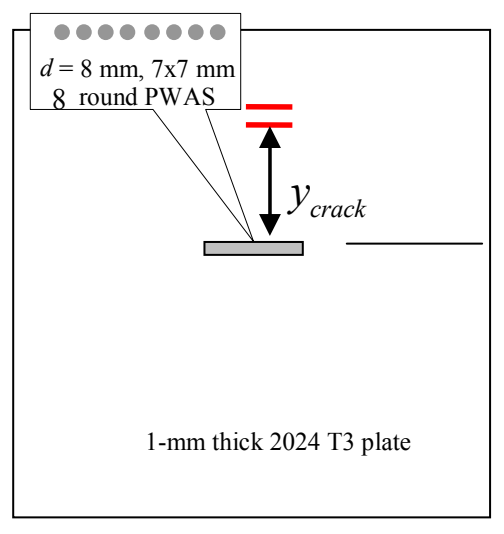

Figure 7 1-D PWAS array, crack and plate setup: (a) case I, II; (b) case III, IV

Figure 8 shows the EUSR scanning images and $90^{\circ}$ A-scan signals of one broadside crack in case I, located at $200 \mathrm{~mm} / 90^{\circ}$ and detected with $36 \mathrm{kHz}$ A0 mode. Without dispersion compensation, the EUSR scanning image is big and blur (Figure 8a) because its A-scan signal at $90^{\circ}$ spreads out in time domain 
(Figure 8c). In contrast, after dispersion compensation, the scanning image becomes sharper (Figure 8b) and its A-scan signal at $90^{\circ}$ is compressed (Figure 8d). Similar improvements in EUSR scanning images were observed when using $350 \mathrm{kHz}$ S0 mode to detect a crack in case II with the aid of dispersion compensation (Figure 9).

Figure 10 demonstrates the EUSR inspection results of two broadside cracks (case III) distanced by $20 \mathrm{~mm}$ using $350 \mathrm{kHz}$ S0 mode with and without dispersion compensation. In both cases, these two cracks were detected. However a higher contrast ratio of scanning image (Figure 10b) was obtained after using dispersion compensation. When the two cracks on plate are separated only by $16 \mathrm{~mm}$ (case IV), EUSR lost its resolution without dispersion compensation, i.e., only one crack was observed in canning image (Figure 11a). However, after applying dispersion compensation, both of the cracks were detected (Figure 11b).
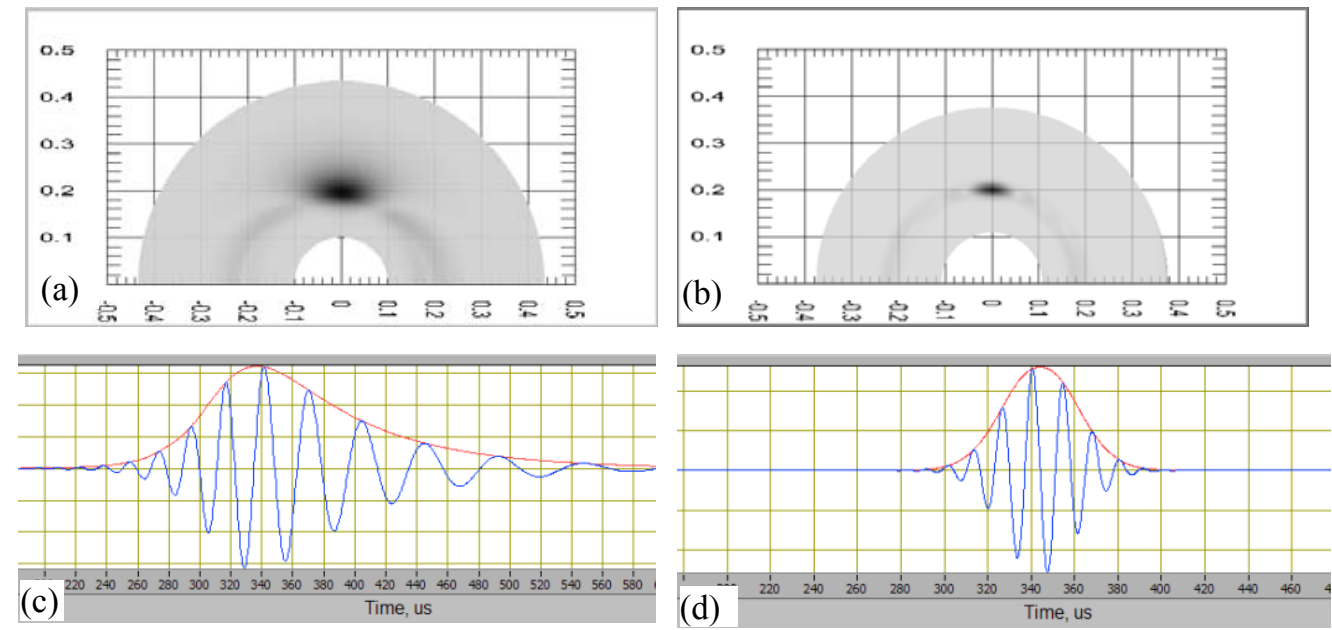

Figure 8 EUSR inspection results for case I (3-count 36kHz A0 mode, crack located at $x=200 \mathrm{~mm}$ ) : (a) mapped EUSR scanning image without dispersion compensation; (b) mapped EUSR scanning image with dispersion compensation; (c) selected A-scan at $90^{\circ}$ without dispersion compensation; (d) selected A-scan at $90^{\circ}$ with dispersion compensation.
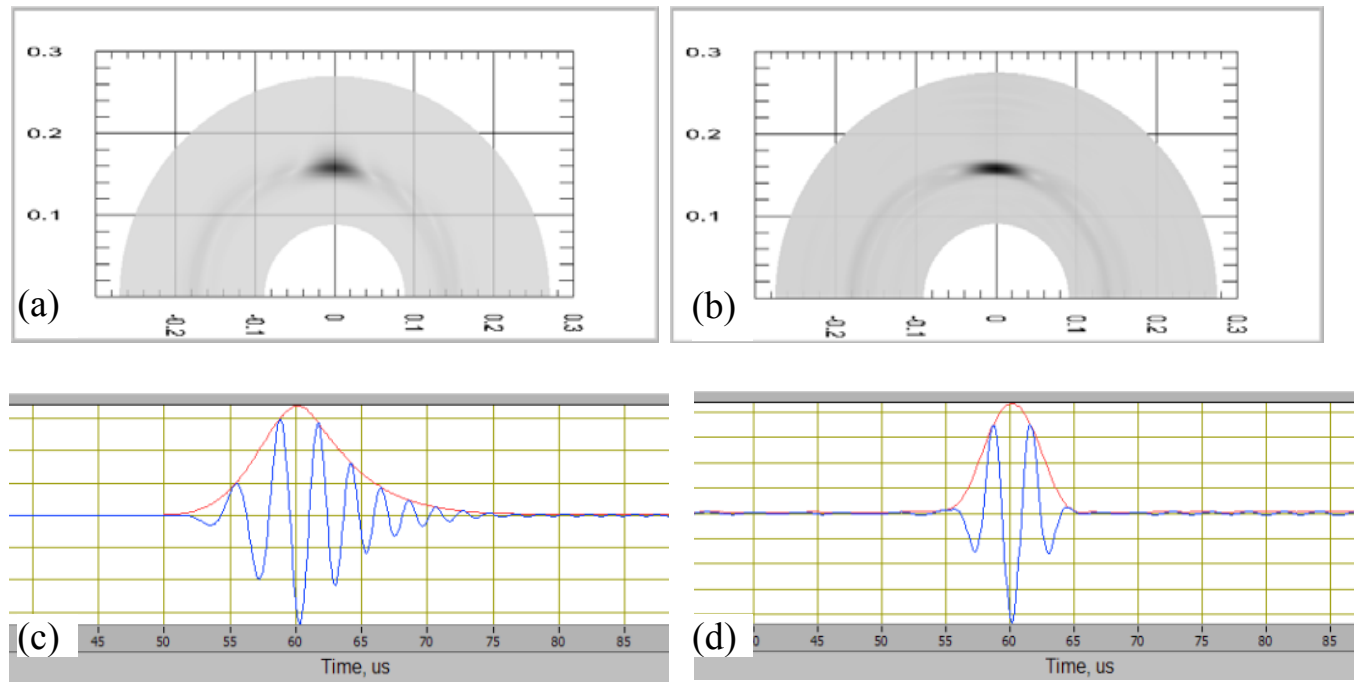

Figure 9 EUSR inspection results for case II (3.5-count $350 \mathrm{kHz}$ S0 mode, crack located at $x$ $=150 \mathrm{~mm}$ ) : (a) mapped EUSR scanning image without dispersion compensation; (b) mapped EUSR scanning image with dispersion compensation; (c) selected A-scan at $90^{\circ}$ without dispersion compensation; (d) selected A-scan at $90^{\circ}$ with dispersion compensation. 

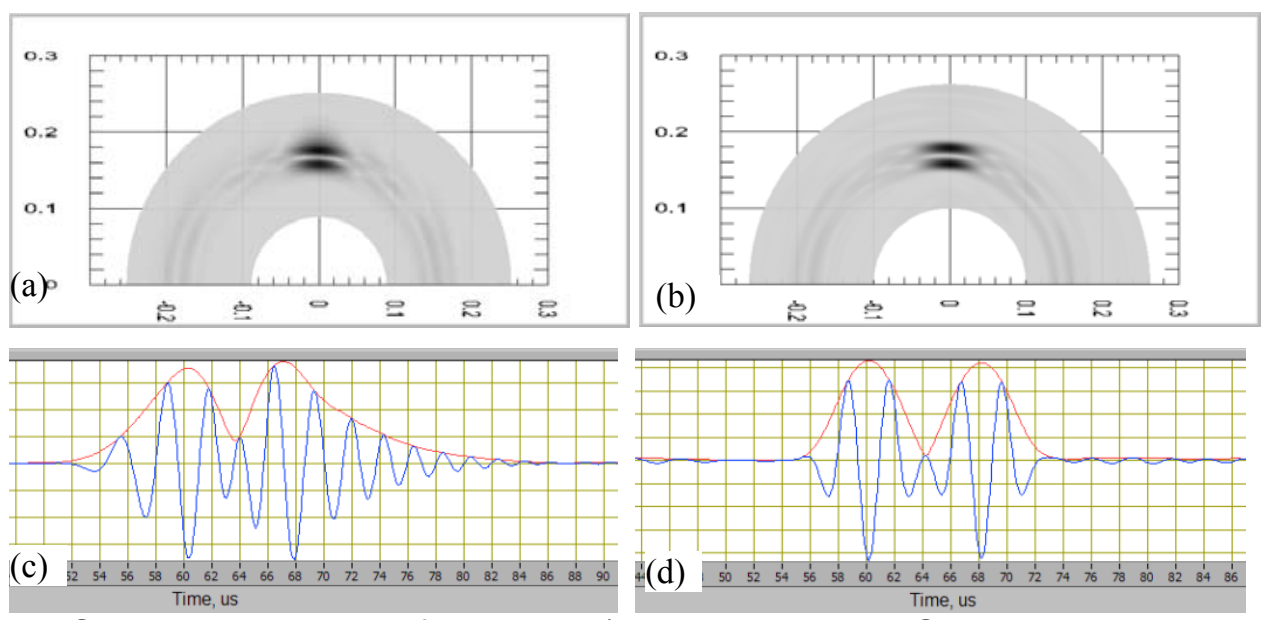

Figure 10 EUSR inspection results for case III (3.5-count $350 \mathrm{kHz}$ S0 mode, two cracks located at $\mathrm{x}$ $=150 \mathrm{~mm}$ and $x=170 \mathrm{~mm}$ ) : (a) mapped EUSR scanning image without dispersion compensation; (b) mapped EUSR scanning image with dispersion compensation; (c) selected A-scan at $90^{\circ}$ without dispersion compensation; (d) selected A-scan at $90^{\circ}$ with dispersion compensation.
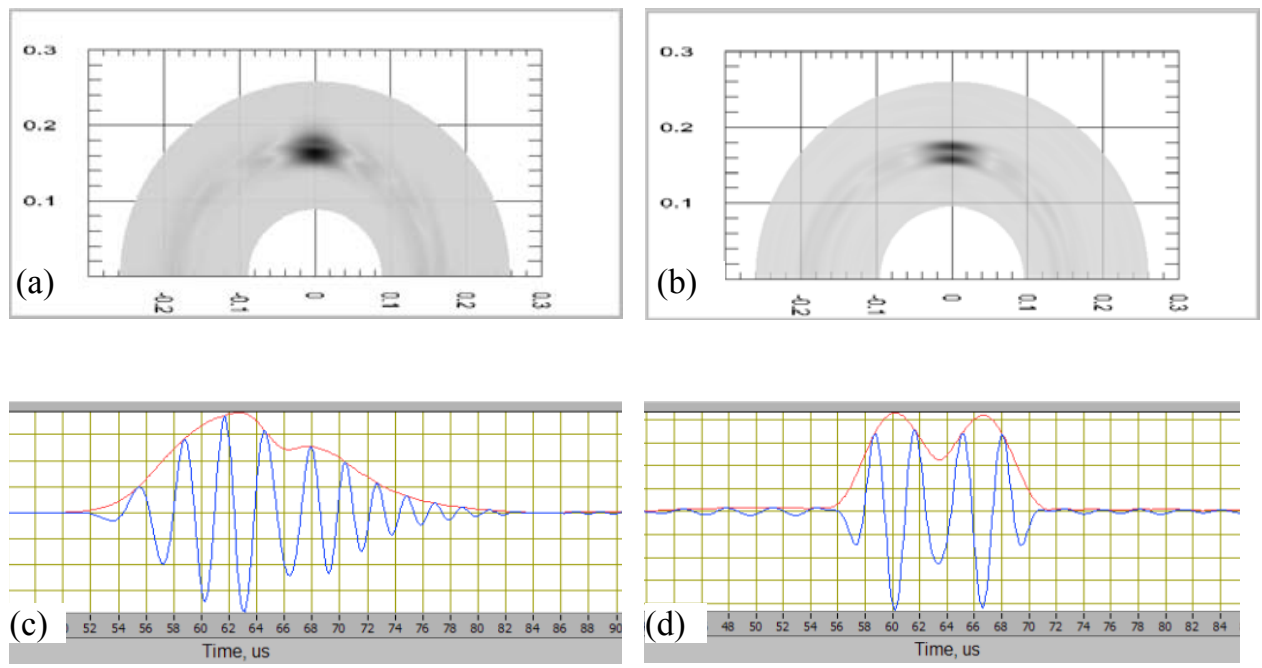

Figure 11 EUSR inspection results for case IV (3.5-count $350 \mathrm{kHz}$ S0 mode, two cracks located at $x$ $=150 \mathrm{~mm}$ and $\mathrm{x}=166 \mathrm{~mm}$ ): (a) mapped EUSR scanning image without dispersion compensation; (b) mapped EUSR scanning image with dispersion compensation; (c) selected A-scan at $90^{\circ}$ without dispersion compensation; (d) selected A-scan at $90^{\circ}$ with dispersion compensation.

\section{CONCLUSIONS}

Nonlinear characteristic of the wavenumber is the cause of the Lamb wave dispersion. The dispersion causes the elongation of received waves, deteriorates the spatial resolution of the waves, makes the experimental data hard to be interpreted, and limits the selection of Lamb wave operation frequency.

In this paper, dispersion compensation and removal algorithms were first theoretically investigated and compared for recovering S0 and A0 mode Lamb waves using both simulation and experimental data. It was found that both algorithms were able to well recover the original shape of a dispersed S0 wave packet. By mapping a wave packet from time domain to spatial domain, the dispersion compensation algorithm is also able to directly recover a wave packet's spatial location, which is not available in the dispersion removal algorithm. However, the dispersion removal algorithm outperformed the dispersion compensation algorithm for recovering the A0 mode Lamb wave. Moreover, the dispersion removal 
algorithm takes less computation efforts than the dispersion compensation algorithm. Next, the dispersion compensation algorithm was applied to EUSR PWAS phased array to image a single crack and two closely located cracks on a plate. From the simulation, we conclude that the dispersion compensation can help improve the EUSR spatial resolution. In the presented EUSR dispersion compensation simulation, the compensation algorithm was applied to transducers' raw data directly, application of the algorithm to the A-scan data is suggested for future work to save computation effort.

\section{ACKNOWLEDGMENTS}

The financial support of National Science Foundation under Grant \#CMS-0408578 is thankfully acknowledged.

\section{REFERENCES}

Giurgiutiu, V.; Bao, J. (2002) "Embedded-Ultrasonics Structural Radar for the Nondestructive Evaluation of ThinWall Structures" Proceedings of the 2002 ASME International Mechanical Engineering Congress, November 17-22, 2002, New Orleans, LA, paper \# IMECE2002-39017, CD-ROM

Giurgiutiu, V. (2005) "Tuned Lamb-Wave Excitation and Detection with Piezoelectric Wafer Active Sensors for Structural Health Monitoring”, Journal of Intelligent Material Systems and Structures, Vol. 16, No. 4, pp. 291-306

Liu, L.; Yuan, F.G. (2009) “A Linear Mapping Technique for Dispersion Removal of Lamb Waves”, SHM Journal, in Review

Sachse W.; Pao Y.H. (1978) "On the Determination of Phase and Group Velocities of Dispersive Waves in Solids", Journal of Applied Physics, 49(8), August, 1978

Santoni, G. B.; Yu, L.; Xu, B.; Giurgiutiu, V. (2007) "Lamb Wave Mode Tuning of Piezoelectric Wafer Active Sensors for Structural Health Monitoring", ASME Journal of Vibration and Acoustics, Vol. 129, No. 6, pp. 752-762, Dec. 2007

Wilcox, P. D.; Lowe, M. J. S.; Cawley, P. (2001), "A signal processing technique to remove the effect of dispersion from guided wave signals," in Review of Progress in Quantitative Nondestructive Evaluation, Vol. 20, D. O. Thompson and D. E. Chimenti, Eds. New York: AIP Conference Proceedings, 2001, pp. 555-562.

Wilcox, P. (2003), "A Rapid Signal Processing Technique to Remove the Effect of Dispersion from Guided Wave Signals", IEEE Transactions on Ultrasonics, Ferroelectrics, and Frequency Control, Vol. 50, No. 4, pp. 419-427

Xu, B.; Giurtiutiu, V., 2007, "Single Mode Tuning Effects on LambWave Time Reversal with Piezoelectric Wafer Active Sensors for Structural Health Monitoring", Journal of Nondestructive Evaluation, Vol. 26, pp. $123 \sim 134$

Yu, L.; Giurgiutiu, V. (2007) "In-situ Optimized PWAS Phased Arrays for Lamb Wave Structural Health Monitoring", Journal of Mechanics of Materials and Structures, Vol.2, No.3, pp. 459-488, 